\title{
ANÁLISIS DE UN SISTEMA DE PETROVSKY ABSTRACTO VÍA EDO
}

\section{Víctor Rafael Cabanillas Zannini ${ }^{1}$}

RESUMEN.- Estudiamos el problema de evolución abstracto

$$
\left\{\begin{array}{l}
\frac{d^{2} u}{d t^{2}}+\delta \frac{d u}{d t}+A^{\alpha} u+p(t) A^{\beta} u=f \\
u(0)=u_{0}, \frac{d u}{d t}(0)=u_{1}
\end{array}\right.
$$

el cual es una generalización de un sistema linealizado de Petrovsky con disipación.

Mostramos que es posible obtener información sobre la regularidady decaimiento de (*) a partir del estudio del sistema de ecuaciones diferenciales ordinarias (EDO) asociado a (*) mediante técnicas de EDO.

PALABRAS CLAVE.- Ecuación de evolución ajstracta. Desigualdad de Gronwall. Identidad de energía. Decaimiento de soluciones.

\section{ANALYSIS OF AN ABSTRACT PETROVSKY'S SYSTEM VIA EDO}

ABSTRACT.- We study the problem of abstract evolution

$$
\left\{\begin{array}{l}
\frac{d^{2} u}{d t^{2}}+\delta \frac{d u}{d t}+A^{\alpha} u+p(t) A^{\beta} u=f \\
u(0)=u_{0}, \frac{d u}{d t}(0)=u_{1}
\end{array}\right.
$$

which is a generalization of a linearized system of Petrovsky with dissipative.

We show that is possible to obtain information on the regularity and decay of the given system, from the study of the system of ordinary differential equations (ODE) associated to the system (*) by means of ODE techniques.

KEYWORDS.- Equations of abstract evolution. Gronwall's inequality. Energy identity. Decay of solutions.

\footnotetext{
${ }^{1}$ Universidad Nacional Mayor.de San Marcos. Facultad de Ciencias Matemáticas.e-mail: vcabanillasz@unmsmedu.pe
} 


\section{Introducción}

Es bien conocido que si $P, Q, c:[0, T] \rightarrow \mathbb{R}$ son tres funciones continuas, entonces el problema de valor inicial (PVI)

$$
\left\{\begin{array}{l}
y^{\prime \prime}+P(t) y^{\prime}+Q(t) y=c(t) \\
y(0)=y_{0}, \quad y^{\prime}(0)=y_{1}
\end{array}\right.
$$

admite una única solución en el intervalo $[0, T]$, siendo $y^{\prime}=\frac{d y}{d t}$, además $y_{0}, y_{1}$ son dos números reales arbitrarios dados.

En este artículo estudiamos un PVI del tipo $(1.1) \operatorname{con} P(t) \equiv \delta, Q(t)=\lambda^{\beta}\left(\lambda^{\alpha-\beta}+P(t)\right)$, i.e.,

$$
\left\{\begin{array}{l}
y^{\prime \prime}+\delta y^{\prime}+\lambda^{\beta}\left(\lambda^{\alpha-\beta}+p(t)\right) y=c(t) \\
y(0)=y_{0} \quad, \quad y^{\prime}(0)=y_{1}
\end{array}\right.
$$

siendo $\delta, \alpha, \beta, \lambda \in \mathbb{R}^{+}$y $p:[0, T] \rightarrow \mathbb{R}$ una función continua, además $c$ es una función medible y acotada.

Nuestro interés por este problema surge del estudio del problema de evolución abstracto

$$
\left\{\begin{array}{l}
\frac{d^{2} u}{d t^{2}}+\delta \frac{d u}{d t}+A^{\alpha} u+p(t) A^{\beta} u=f \\
u(0)=u_{0}, \frac{d u}{d t}(0)=u_{1}
\end{array}\right.
$$

donde $A$ es un operador en un espacio de Hilbert $H$ y $f$ es una función medible y acotada con valores en $H$. Los vectores $u_{0}$ y $u_{1}$ también son dados en $H$.

Una manera clásica de abordar el problema de Cauchy (1.3) es por medio del Método de Faedo-Galerkin a través de estimativas sobre la solución $u_{m}$ del problema aproximado asociado a (1.3), i.e.,

$$
\left\{\begin{array}{l}
\left(u_{m}^{\prime \prime}(t)+\delta u_{m}^{\prime}(t)+A^{\alpha} u_{m}(t)+p(t) A^{\beta} u_{m}(t)-f(t), w_{k}\right)=0 \\
\left(u_{m}(0), w_{k}\right)=u_{0 m},\left(u_{m}^{\prime}(0), w_{k}\right)=u_{1 m}
\end{array}\right.
$$

donde $u_{m}$ se denomina solución aproximada, y $\left\{w_{k}\right\}_{k \in \mathbb{N}}$ es una base adecuada de $H$. Tal como se mostrará en la sección 4, la ecuación (1.4) nos lleva a estudiar un PVI de la forma

$$
\left\{\begin{array}{l}
g_{k}^{\prime \prime}(t)+\delta g_{k}^{\prime}(t)+\lambda_{k}^{\alpha} g_{k}(t)+\lambda_{k}^{\beta} p(t) g_{k}(t)=\left(f(t), w_{k}\right) \\
g_{k}(0)=\left(u_{0}, w_{k}\right), \quad g_{k}^{\prime}(0)=\left(u_{1}, w_{k}\right)
\end{array}\right.
$$

el cual tiene la forma (1.2), siendo $\left\{\lambda_{k}\right\}_{k \in \mathbb{N}}$ la sucesión de autovalores del operador $A$ asociada a la base $\left\{w_{k}\right\}_{k \in \mathbb{N}}$. 
Por medio de un teorema de existencia y unicidad para la EDO (1.2) garantizamos que (1.5) admite solución única, con lo cual (1.4) también admite única solución para cada $m \in \mathbb{N}$. Una vez garantizada la existencia de solución hacemos estimativas a priori sobre la sucesión $\left\{u_{m}\right\}_{m \in \mathbb{N}}$ y la sucesión $\left\{u_{m}^{\prime}\right\}_{m \in \mathbb{N}}$ de sus derivadas, para así poder obtener un candidato a solución, el cual se obtiene como el límite débil de una subsucesión de $\left\{u_{m}\right\}_{m \in \mathbb{N}}$. La etapa final consiste en probar, por un proceso límite, que el candidato a solución es, en efecto, la solución que buscamos. Esto describe, a grosso modo, el Método de Faedo-Galerkin.

Al estudiar (1.3) observamos que es posible tomar un "atajo" y economizar buena parte de los cálculos y estimativas que implicaria trabajar con el clásico Método de Faedo-Galerkin. El "atajo" consiste en explorar las propiedades de la EDO (1.5) asociada al problema aproximado (1.4). Mediante la aplicación de desigualdades y métodos de EDO logramos obtener una acotación de la solución $g_{k}$ de (1.5) así como de su derivada $g_{k}^{\prime}$, esta acotación nos permite obtener el candidato a solución del problema abstracto (1.3). La ventaja de esta técnica, además de simplificar los cálculos, es que permite obtener directamente una solución $u$ de (1.3) bastante regular, a saber $u \in C\left([0, T], D\left(A^{\frac{\alpha}{2}}\right)\right) \cap C^{\mathrm{l}}([0, T] ; H)$. Además, los métodos y técnicas expuestos en este artículo son bastante simples y pueden ser aplicados a una variedad de problemas.

El resto del artículo está organizado de la siguiente manera: en la sección 2 garantizamos la existencia de una solución local del PVI (1.2) y obtenemos una estimativa que permite extender o prolongar las soluciones al intervalo deseado $[0, T]$, para $T>0$ arbitrario. La sección 3 está dedicada a la obtención de una estimativa de decaimiento exponencial para el PVI (1.2). Los resultados de las secciones 2 y 3 se resumen en el Teorema 3.3. En la sección 4 fijamos las hipótesis para el estudio del problema abstracto (1.1) y reseñamos algunos casos particulares de (1.3). La existencia y unicidad de solución del problema (1.3) así como el teorema central del trabajo son mostrados en la sección 5. Finalmente, en la sección 6 indicamos algunas posibles extensiones de este artículo.

\section{Existencia y Extensión de las Soluciones}

Consideremos la ecuación diferencial ordinaria de segundo orden

$$
\left\{\begin{array}{l}
y^{n}+\delta y^{\prime}+\lambda^{\beta} y+\lambda^{\beta} p(t) y=c(t) \\
y(0)=y_{0}, \quad y^{\prime}(0)=y_{1}
\end{array}\right.
$$

donde $\delta, \lambda, \alpha$ y $\beta$ son números reales positivos. Dado $T>0$, si $p$ es una función continua en $[0, T]$ y $c$ es una función medible y acotada, el PVI (2.1) admite una única solución local $u$ definida sobre un intervalo $\left[0, t_{0}\right)$. Con el fin de extender esta solución al intervalo $[0, T]$ debemos hacer algunas estimativas. Veamos:

Multiplicando la ecuación (2.1) por $y^{\prime}$ obtenemos

$$
\frac{1}{2} \frac{d}{d t}\left[\left(y^{\prime}\right)^{2}+\lambda^{\alpha} y^{2}\right]+\delta\left(y^{\prime}\right)^{2}=c(t) y^{\prime}-\lambda^{\beta} p(t) y y^{\prime}
$$




$$
\left\{\begin{array}{l}
-\lambda^{\beta} p(t) y y^{\prime} \leq \frac{\lambda^{\beta-\frac{\alpha}{2}}}{2} \max _{t \in[0, T]}|p(t)|\left(\left(y^{\prime}\right)^{2}+\lambda^{\alpha} y^{2}\right) \\
c(t) y^{\prime} \leq \delta\left(y^{\prime}\right)^{2}+\frac{1}{4 \delta} c(t)^{2}
\end{array}\right.
$$

Integrando (2.2) de 0 a $t$ y aplicando (2.3) tenemos

$$
\begin{gathered}
\frac{1}{2}\left(y^{\prime}(t)^{2}+\lambda^{\alpha} y(t)^{2}\right)+\lambda \int_{0}^{t} y^{\prime}(s)^{2} d s \leq \int_{0}^{t}\left(\delta y^{\prime}(s)^{2}+\frac{1}{4 \delta} c(s)^{2}\right) d s+ \\
+\frac{\lambda^{\alpha-\frac{\alpha}{2}}}{2} m_{0} \int_{0}^{t}\left(y^{\prime}(s)^{2}+\lambda^{\alpha} y(s)^{2}\right) d s+\frac{1}{2}\left(y_{1}^{2}+\lambda^{\alpha} y_{0}^{2}\right)
\end{gathered}
$$

donde $m_{0}=\max _{t \in[0, T]}|p(t)|$. Luego,

$$
y^{\prime}(t)^{2}+\lambda^{\alpha} y(t)^{2} \leq y_{1}^{2}+\lambda^{\alpha} y_{0}^{2}+\frac{1}{2 \delta} \int_{0}^{t} c(s)^{2} d s+\lambda^{\beta-\frac{\alpha}{2}} m_{0} \int_{0}^{t}\left(y^{\prime}(s)+\lambda^{\alpha} y(s)^{2}\right) d s
$$

Una aplicación de la Desigualdad de Gronwall en esta última desigualdad nos permite concluir que

$$
y^{\prime}(t)^{2}+\lambda^{\alpha} y(t)^{2} \leq\left(y_{1}^{2}+\lambda^{\alpha} y_{0}^{2}+\frac{1}{2 \delta} \int_{0}^{t} c(s)^{2} d s\right) \exp \left(\lambda^{\beta-\frac{\alpha}{2}} m_{0} t\right)
$$

La desigualdad (2.4) nos permite probar que la solución $y$ de (2.1) puede ser extendida o prolongada al intervalo $[0, T]$ para $T>0$ arbitrario (ver [4]).

Debido a que (2.1) posee solución en el intervalo $[0, T]$, siendo $T$ un número real arbitrario, es natural preguntarnos ¿qué sucede con la solución $y=y(t)$ para tiempos $t$ muy grandes? La estimativa (2.4) no brinda esta información, pues cuando $t$ es muy grande el término exponencial hace que el segundo miembro tienda para infinito. Entonces, es necesario otro tipo de estimativa. En la siguiente sección buscaremos obtener información acerca del comportamiento asintótico de las soluciones de (2.1) cuando $c \equiv 0$.

Observación 2.1 (Dependencia Contínua) Fijado $T>0$, de (2.4) tenemos que

$$
y^{\prime}(t)^{2}+\lambda^{\alpha} y(t)^{2} \leq\left(y_{1}^{2}+\lambda^{\alpha} y_{0}^{2}+\frac{1}{2 \delta} \int_{0}^{T} c(s)^{2} d s\right) \exp \left(\lambda^{\beta-\frac{g}{2}} m_{0} T\right)
$$

es decir, existe una solución $K>0$ tal que

$$
|y(t)|^{2}+\left|y^{\prime}(t)\right|^{2} \leq K\left(\left|y_{0}\right|^{2}+\left|y_{1}\right|^{2}+\|c\|_{L^{2}(0, T)}^{2}\right)
$$


Esta desigualdad muestra que la solución $y$, así como su derivada dependen continuamente de $c$ y de las condiciones inciales $y_{0}, y_{1}$.

\section{Una Estimativa de Decaimiento}

Consideremos el sistema homogéneo asociado a (2.1), es decir

$$
\left\{\begin{array}{l}
y^{\prime \prime}+\delta y^{\prime}+\lambda^{\alpha} y+\lambda^{\beta} p(t) y=0 \\
y(0)=y_{0}, \quad y^{\prime}(0)=y_{1}
\end{array}\right.
$$

Definamos la función $\varphi:[0, T] \rightarrow \mathbb{R}$ de la siguiente manera

$$
\varphi(t)=\frac{1}{2}\left(y^{\prime}(t)^{2}+\lambda^{\beta}\left(\lambda^{\alpha-\beta}+p(t)\right) y(t)^{2}\right)+\frac{\delta}{2}\left(y(t) y^{\prime}(t)+\frac{\delta}{2} y(t)^{2}\right)
$$

Notemos que

$$
-\frac{1}{2 \delta} y^{\prime}(t)^{2}-\frac{\delta}{2} y(t)^{2} \leq y(t) y^{\prime}(t) \leq \frac{1}{2 \delta} y^{\prime}(t)^{2}+\frac{\delta}{2} y(t)^{2}
$$

Aplicando esta desigualdad a (3.2) tenemos

$$
\begin{aligned}
\varphi(t) & \leq \frac{1}{2}\left(y^{\prime}\right)^{2}+\frac{1}{2} \lambda^{\alpha} y^{2}+\frac{1}{2} \lambda^{\beta} p(t) y^{2}+\frac{\delta}{2}\left(\frac{1}{2 \delta}\left(y^{\prime}\right)^{2}+\frac{\delta}{2} y^{2}\right)+\frac{\delta^{2}}{4} y^{2} \\
& =\frac{3}{4}\left(\left(y^{\prime}\right)^{2}+\lambda^{\alpha} y^{2}\right)-\frac{1}{4} \lambda^{\alpha} y^{2}+\frac{1}{2} \lambda^{\beta} p(t) y^{2}+\frac{\delta^{2}}{2} y^{2}
\end{aligned}
$$

es decir

$$
\varphi(t) \leq \frac{3}{4}\left(\left(y^{\prime}(t)\right)^{2}+\lambda^{\alpha} y(t)^{2}\right)-\frac{1}{2}\left[\lambda^{\beta}\left(\frac{\lambda^{\alpha-\beta}}{2}-p(t)\right)-\delta^{2}\right] y(t)^{2}
$$

Entonces si $\lambda^{\beta}\left(\frac{\lambda^{\alpha-\beta}}{2}-p(t)\right)-\delta^{2} \geq 0$, tenemos

$$
\varphi(t) \leq \frac{3}{4}\left(\left(y^{\prime}\right)^{2}+\lambda^{\alpha} y^{2}\right)
$$

Por otra parte, si aplicamos la primera desigualdad de (3.3) a (3.2) obtenemos

$$
\begin{aligned}
\varphi(t) & \geq \frac{1}{2}\left(y^{\prime}\right)^{2}+\frac{1}{2} \lambda^{\alpha} y^{2}+\frac{1}{2} \lambda^{\beta} p(t) y^{2}+\frac{\delta}{2}\left(-\frac{1}{2 \delta}\left(y^{\prime}\right)^{2}-\frac{\delta}{2} y^{2}\right)+\frac{\delta^{2}}{4} y^{2} \\
& =\frac{1}{4}\left(\left(y^{\prime}\right)^{2}+\lambda^{\alpha} y^{2}\right)+\frac{1}{2} \lambda^{\beta}\left(\frac{\lambda^{\alpha-\beta}}{2}+p(t)\right) y^{2}
\end{aligned}
$$


entonces si $\frac{\lambda^{\alpha-\beta}}{2}+p(t) \geq 0$ se tiene

$$
\varphi(t) \geq \frac{1}{4}\left(\left(y^{\prime}\right)^{2}+\lambda^{\alpha} y^{2}\right)
$$

De (3.4) y (3.5) tenemos que si

$$
\lambda^{\beta}\left(\frac{\lambda^{\alpha-\beta}}{2}-p(t)\right)-\delta^{2} \geq 0 \text { y } \frac{\lambda^{\alpha-\beta}}{2}+p(t) \geq 0
$$

entonces

$$
\frac{1}{4}\left(y^{\prime}(t)^{2}+\lambda^{\alpha} y(t)^{2}\right) \leq \varphi(t) \leq \frac{3}{4}\left(y^{\prime}(t)^{2}+\lambda^{\alpha} y(t)^{2}\right)
$$

Observación 3.1 Notemos que las condiciones en (3.6) pueden resumirse en la siguiente condición

$$
\frac{\lambda^{\alpha-\beta}}{2} \leq p(t) \leq \frac{\lambda^{\alpha-\beta}}{2}-\lambda^{-\beta} \delta^{2}
$$

Nuestro objetivo es obtener estimativas que nos indiquen acerca del comportamiento de la solucón $y$ del PVI homogéneo (3.1) para tiempos muy grandes.

Derivando la función $\varphi$ tenemos

$$
\frac{d}{d t} \varphi=y^{\prime} y^{\prime \prime}+\lambda^{\alpha} y y^{\prime}+\frac{1}{2} \lambda^{\beta} p^{\prime}(t) y^{2}+\lambda^{\beta} p(t) y y^{\prime}+\frac{\delta}{2}\left(y^{\prime}\right)^{2}+\frac{\delta}{2} y\left(y^{\prime \prime}+\delta y^{\prime}\right)
$$

Pero y es solución de (3.1) entonces satisface $y^{n}+\delta y^{\prime}=-\lambda^{\alpha} y-\lambda^{\beta} p(t) y$. Reemplazando en la igualdad anterior tenemos

$$
\begin{gathered}
\frac{d}{d t} \varphi=y^{\prime} y^{\prime \prime}+\lambda^{\alpha} y y^{\prime}+\frac{1}{2} \lambda^{\beta} p^{\prime}(t) y^{2}+\lambda^{\beta} p(t) y y^{\prime}+\frac{\delta}{2}\left(y^{\prime}\right)^{2}-\frac{\delta}{2} \lambda^{\beta}\left(\lambda^{\alpha-\beta}+p(t)\right) y^{2}= \\
=y^{\prime}\left(y^{\prime \prime}+\delta y^{\prime}+\lambda^{\alpha} y+\lambda^{\beta} p(t) y\right)-\frac{\delta}{2}\left(y^{\prime}\right)^{2}-\frac{1}{2}\left[\delta \lambda^{\beta}\left(\lambda^{\alpha-\beta}+p(t)\right)-\lambda^{\beta} p^{\prime}(t)\right] y^{2}
\end{gathered}
$$

entonces por (3.1) se tiene que

$$
\frac{d}{d t} \varphi(t)=-\frac{\delta}{2} y^{\prime}(t)^{2}-\frac{1}{2}\left[\delta \lambda^{\beta}\left(\lambda^{\alpha-\beta}+p(t)\right)-\lambda^{\beta} p^{\prime}(t)\right] y(t)^{2}
$$

De esta última identidad y aplicando (3.7) tenemos

$$
\begin{aligned}
\frac{d}{d t} \varphi+\frac{\delta}{2} \varphi & \leq-\frac{\delta}{2}\left(y^{\prime}\right)^{2}-\frac{1}{2}\left[\delta \lambda^{\beta}\left(\lambda^{\alpha-\beta}+p(t)\right)-\lambda^{\beta} p^{\prime}(t)\right] y^{2}+\frac{3 \delta}{8}\left(\left(y^{\prime}\right)^{2}+\lambda^{\alpha} y^{2}\right) \\
& =-\frac{\delta}{8}\left(y^{\prime}\right)^{2}-\frac{\lambda^{\beta}}{2}\left(\delta\left(\frac{\lambda^{\alpha-\beta}}{4}+p(t)\right)-p^{\prime}(t)\right) y^{2}
\end{aligned}
$$


Entonces si

$$
\delta\left(\frac{\lambda^{\alpha-\beta}}{4}+p(t)\right)-p^{\prime}(t) \geq 0
$$

tenemos que

$$
\frac{d}{d t} \varphi(t)+\frac{\delta}{2} \varphi(t) \leq 0
$$

de donde, aplicando la desigualdad de Gronwall obtenemos

$$
\varphi(t) \leq \varphi(0) \exp \left(-\frac{\delta}{2} t\right)
$$

Aplicando una vez más la desigualdad (3.7) llegamos a

$$
y^{\prime}(t)^{2}+\lambda^{\alpha} y(t)^{2} \leq 3\left(y_{1}^{2}+\lambda^{\alpha} y_{0}^{2}\right) \exp \left(-\frac{\delta}{2} t\right)
$$

Observación 3.2 (Decaimiento Exponencial) La estimativa (3.12) responde a nuestra pregunta sobre el comportamiento de las soluciones del problema homogéneo asociado a (2.1) para tiempos muy grandes. El término exponencial del segundo miembro de (3.12) indica que a medida que $t$ crece, la solución $y$ de (2.1) así como su derivada $y^{\prime}$ decrecen exponencialmente, es decir, decaen exponencialmente.

Podemos resumir lo expuesto en estas dos últimas secciones en el siguiente

Teorema 3.3 Supongamos que $\delta, \alpha, \beta$ y $\lambda$ son constantes positivas y sean $p$ una función continua y c una función medible acotada sobre $[0, T]$, para $T>0$ dado. Entonces el problema de valor inicial (2.1) admite una única solución el intervalo $[0, T]$. Esta solución satisface la siguiente desigualdad

$$
y^{\prime}(t)^{2}+\lambda^{\alpha} y(t)^{2} \leq\left(y_{1}^{2}+\lambda^{\alpha} y_{0}^{2}+\frac{1}{2 \delta} \int_{0}^{t} c(s)^{2} d s\right) \exp \left(\lambda^{\beta-\frac{\alpha}{2}} m_{0} t\right)
$$

para todo $t \in[0, T]$, donde $m_{0}=\max _{t \in[0, T]}|p(t)|$. Además si $c \equiv 0$ y $p \in C^{1}([0, T])$ es tal que para todo $t \in[0, T]$ satisface

$$
\left\{\begin{array}{l}
\frac{\lambda^{\alpha-\beta}}{2} \leq p(t) \leq \frac{\lambda^{\alpha-\beta}}{2}-\lambda^{-\beta} \delta^{2} \\
\delta\left(\frac{\lambda^{\alpha-\beta}}{4}+p(t)\right)-p^{\prime}(t) \geq 0
\end{array}\right.
$$

entonces se tiene la siguiente estimativa de decaimiento

$$
y^{\prime}(t)^{2}+\lambda^{\alpha} y(t)^{2} \leq 3\left(y_{1}^{2}+\lambda^{\alpha} y_{0}^{2}\right) \exp \left(-\frac{\delta}{2} t\right)
$$




\section{El Problema Abstracto Asociado a (2.1)}

El Problema de Cauchy (2.1) que hemos estudiado en las secciones anteriores aparece en el estudio del siguiente problema abstracto

$$
\left\{\begin{array}{l}
\frac{d^{2} u}{d t^{2}}+\delta \frac{d u}{d t}+A^{\alpha} u+p(t) A^{\beta} u=f \\
u(0)=u_{0}, \frac{d u}{d t}(0)=u_{1}
\end{array}\right.
$$

En (4.1) $f$ es una función vectorial definida sobre $(0, T)$ y que asume valores en un espacio de Hilbert $H$; $A$ es un operador definido en $H ; u_{0}$ y $u_{1}$ son dados en $H$ o en subespacios de $H$ y representan las condiciones iniciales. Además, supondremos que $\alpha \geq 2 \beta>0$.

El PVI (4.1) es un problema de evolución abstracto que, dependiendo del operador $A$, describe una variedad de fenómenos físicos. Por ejemplo, si $A=-\Delta, \alpha=1, \delta=0$ y $p=0$ tenemos la ecuación de las ondas

$$
\frac{\partial^{2} u}{\partial t^{2}}-\Delta u=f
$$

la misma que con una condición de frontera de Dirichlet homogénea, i.e.,

$$
\begin{cases}\frac{\partial^{2} u}{\partial t^{2}}-\Delta u=f & , \text { en } \Omega \times R^{+} \\ u=0 & , \text { sobre } \partial \Omega \times R^{+} \\ u(0)=u_{0}, \frac{\partial u}{\partial t}(0)=u_{1} & , \text { en } \Omega\end{cases}
$$

describe las vibraciones transversales de una membrana $\Omega$ con configuración inicial $u_{0}$ y velocidad incial $u_{1}$. Si $\delta \neq 0$ tenemos la ecuación de ondas con disipación

$$
\frac{\partial^{2} u}{\partial t^{2}}+\delta \frac{\partial u}{\partial t}-\Delta u=f
$$

Si $A=-\Delta, \alpha=2, \delta=0$ y $p, f \equiv 0$ tenemos el sistema de Petrovsky

$$
\begin{cases}\frac{\partial^{2} u}{\partial t^{2}}+\Delta^{2} u=0 & , \text { en } \Omega \times R^{+} \\ u=\frac{\partial u}{\partial v}=0 & , \text { sobre } \partial \Omega \times R^{+} \\ u(0)=u_{0}, \frac{\partial u}{\partial t}(0)=u_{1} & , \text { en } \Omega\end{cases}
$$


el cual describe la vibración de una placa sin ninguna perturbación externa. Una ecuación que describe las oscilaciones lineales de una placa con disipación y ante la presencia de agentes externos es dada por

$$
\frac{\partial^{2} u}{\partial t^{2}}+\delta \frac{\partial u}{\partial t}+\Delta^{2} u+p(t) \Delta u=f
$$

el cual también es un caso particular de (4.1), ver [2].

Un problema más general que no trataremos en este artículo es el modelo para las vibraciones no lineales de una placa elástica con disipación interior, i.e.,

$$
\left\{\begin{array}{l}
\frac{d^{2} u}{d t^{2}}(t)+\delta \frac{d u}{d t}(t)+\Delta^{2} u(t)+h\left(\|\nabla u(t)\|^{2}\right) \Delta u(t)=f(t) \\
u(0)=u_{0}, \frac{d u}{d t}(0)=u_{1}
\end{array}\right.
$$

para un estudio sobre la existencia de solución para este modelo ver [1].

El problema de evolución abstracto (4.1) es en general formulado sobre un espacio de Hilbert $H$ de dimensión infinita. La falta de resultados de existencia y unicidad para ecuaciones sobre este tipo de espacios nos lleva a truncar el problema y reducirlo a un espacio de dimensión finita $V_{m}$. Hecho el estudio del problema sobre el espacio finito-dimensional $V_{m}$ regresamos al problema original por un proceso límite. Esta es la idea central del Método de Faedo-Galerkin.

Sea $H$ un espacio de Hilbert separable, de dimensión infinita con producto interno y norma denotados por (,) y $\|$.$\| respectivamente. Sea A$ un operador positivo, autoadjunto con espectro discreto, definido en $H$.

Denotemos por $\left\{w_{k}\right\}_{k \in \mathbb{N}}$ una base ortonormal de $\mathrm{H}$ formada por los autovectores de $A \mathrm{y}$ $\left\{\lambda_{k}\right\}_{k \in \mathbb{N}}$ la correspondiente sucesión de autovalores. Fijado $m>0$, definimos el espacio

$$
V_{m}=\operatorname{span}\left\{w_{1}, w_{2}, \ldots, w_{m}\right\}
$$

Entonces, estudiaremos el problema (4.1) sobre el espacio $m$-dimensional $V_{m}$. Busquemos una solución $u_{m}$ en $V_{m}$ definida por

$$
u_{m}(t)=\sum_{k=1}^{m} g_{k}(t) w_{k}
$$

que satisfaga la ecuación aproximada

$$
\left\{\begin{array}{l}
\left(u_{m}^{\prime \prime}(t)+\delta u_{m}^{\prime}(t)+A^{\alpha} u_{m}(t)+p(t) A^{\beta} u_{m}(t)-f(t), w_{k}\right)=0 \\
\left(u_{m}(0), w_{k}\right)=\left(u_{0}, w_{k}\right)=u_{0 m},\left(u_{m}^{\prime}(0), w_{k}\right)=\left(u_{1}, w_{k}\right)=u_{1 m}
\end{array}\right.
$$


Dada la ortonormalidad de la base $\left\{w_{k}\right\}_{k \in \mathbb{N}}$, reemplazando (4.2) en (4.3) obtenemos el sistema de ecuaciones diferenciales ordinarias

$$
\left\{\begin{array}{l}
g_{k}^{\prime \prime}(t)+\delta g_{k}^{\prime}(t)+\lambda_{k}^{\alpha} g_{k}(t)+\lambda_{k}^{\beta} p(t) g_{k}(t)=\left(f(t), w_{k}\right) \\
g_{k}(0)=\left(u_{0}, w_{k}\right), g_{k}^{\prime}(0)=\left(u_{1}, w_{k}\right)
\end{array}\right.
$$

Si fijamos k y escribimos $g_{k}=y, \lambda_{k}=\lambda, c(t)=\left(f(t), w_{k}\right),\left(u_{0}, w_{k}\right)=y_{0}$ y $\left(u_{1}, w_{k}\right)=y_{1}$, vemos que la ecuación (4.4) tiene la forma de la ecuación (2.1) estudiada en las ecuaciones anteriores.

Entonces, de acuerdo al Teorema 3.3, para cada $k=1,2, \ldots, m$ podemos encontrar una solución $g_{k}$ de (4.4) que satisface (3.13) y cuando $c \equiv 0$ satisface (3.14). Así, hemos obtenido una sucesión $\left\{u_{m}\right\}_{m \in \mathbb{N}}$ de soluciones aproximadas del problema (4.1) en $[0, T]$.

\section{Solución del Problema Abstracto}

Antes de definir lo que se entiende por solución de (4.1) definamos el espacio

$$
W^{\alpha}(0, T)=\left\{\psi \in L^{2}\left(0, T ; D\left(A^{\frac{a}{2}}\right)\right) ; \psi^{\prime} \in L^{2}(0, T ; H)\right\}
$$

el cual, dotado de la norma

$$
\|\psi\|_{W^{\alpha}(0, T)}=\left(\|\psi\|_{L^{2}\left(0, T ; D\left(A^{\frac{\alpha}{2}}\right)\right)}^{2}+\left\|\psi^{\prime}\right\|_{L^{2}(0, T ; H)}^{2}\right)^{\frac{1}{2}}
$$

es un espacio de Banach. El espacio $W^{\alpha}(0, T)$ está continuamente inmerso en $C([0, T] ; H)$. Ver [5].

Defición 5.1 Se denomina Solución débil de (4.1) sobre $[0, T]$ a una función $u \in W^{\alpha}(0, T)$ que verifica $u(0)=u_{0}, u^{\prime}(0)=u_{1}$ en $H$ y que además satisface la ecuación

$$
\begin{gathered}
-\int_{0}^{T}\left(u^{\prime}(t)+\delta u(t), \psi^{\prime}(t)\right) d t+\int_{0}^{T}\left(A^{\frac{\alpha}{2}} u(t), A^{\frac{\alpha}{2}} \psi(t)\right) d t+ \\
+\int_{0}^{T}\left(p(t) A^{\frac{\beta}{2}} u(t), A^{\frac{\beta}{2}} \psi(t)\right) d t=\left(u_{1}+\delta u_{0}, \psi(0)\right)+\int_{0}^{T}(f(t), \psi(t)) d t
\end{gathered}
$$

para todo $\psi \in W^{\alpha}(0, T)$ tal que $\psi(T)=0$.

Gracias a la ortonormalidad de la base $\left\{w_{k}\right\}_{k \in \mathbb{N}}$ tenemos que la aplicación $\Phi_{m, n}:[0, T] \rightarrow \mathbb{R}$ definida por

$$
\Phi_{m, n}(t)=\left\|u_{m+n}^{\prime}(t)-u_{m}^{\prime}(t)\right\|^{2}+\left\|A^{\frac{\alpha}{2}} u_{m+n}(t)-A^{\frac{\alpha}{2}} u_{m}(t)\right\|^{2}
$$

satisface 


$$
\Phi_{m, n}(t)=\sum_{k=m+1}^{m+n}\left(\left(g_{k}^{\prime}(t)\right)^{2}+\lambda^{\alpha}\left(g_{k}(t)\right)^{2}\right), \forall t \in[0, T]
$$

Luego, siendo $g_{k}$ solución de (4.4) y gracias a la desigualdad (3.13) del Teorema 3.3 tenemos

$$
\Phi_{m, n}(t) \leq \sum_{k=m+1}^{m+n}\left[\left(u_{1}, w_{k}\right)^{2}+\lambda_{k}^{\alpha}\left(u_{0}, w_{k}\right)^{2}+\frac{1}{2 \delta} \int_{0}^{T}\left(f(t), w_{k}\right)^{2} d t\right] \exp \left(\lambda^{\beta-\frac{\alpha}{2}} m_{0} t\right)
$$

siendo $m_{0}=\max _{t \in[0, T]}|p(t)|$

De esta desigualdad vemos que si $m, n \rightarrow+\infty$ entonces $\Phi_{m, n}(t) \rightarrow 0$. Luego $\left\{u_{m}^{\prime}\right\}_{m \in \mathbb{N}}$ y $\left\{A^{\frac{\alpha}{2}} u_{m}\right\}_{m \in \mathbb{N}}$ son sucesiones de Cauchy en $C([0, T] ; H)$ sobre cualquier intervalo $[0, T]$, entonces $\left\{u_{m}\right\}_{m \in \mathbb{N}}$ y $\left\{u_{m}^{\prime}\right\}_{m \in \mathbb{N}}$ serán sucesiones de Cauchy en $C\left([0, T] ; D\left(A^{\frac{a}{2}}\right)\right)$ y $C([0, T] ; H)$ respectivamente. Por lo tanto, existe una función $u \in C^{1}([0, T] ; H)$ tal que

$$
\begin{gathered}
u_{m} \rightarrow u \text { en } C\left([0, T] ; D\left(A^{\frac{\alpha}{2}}\right)\right) \\
u_{m}^{\prime} \rightarrow u^{\prime} \text { en } C([0, T] ; H)
\end{gathered}
$$

Consideremos la ecuación aproximada (4.3) y sea $\psi \in W^{\alpha}(0, T)$ tal que $\psi(T)=0$. Multiplicando escalarmente (4.3) por $\psi(t)$ e integrando tenemos

$$
\begin{gathered}
-\int_{0}^{T}\left(u_{m}^{\prime}(t)+\delta u_{m}(t), \psi^{\prime}(t)\right) d t+\int_{0}^{T}\left(A^{\frac{\alpha}{2}} u_{m}(t), A^{\frac{\alpha}{2}} \psi(t)\right) d t+ \\
+\int_{0}^{T}\left(p(t) A^{\frac{\beta}{2}} u_{m}(t), A^{\frac{\beta}{2}} \psi(t)\right) d t=\left(u_{1 m}+\delta u_{0 m}, \psi(0)\right)+\int_{0}^{T}(f(t), \psi(t)) d t
\end{gathered}
$$

Como $\alpha \geq \beta$ entonces $D\left(A^{\alpha}\right) \subset D\left(A^{\beta}\right)$, siendo esta inclusión continua y compacta. Entonces de (5.4) tenemos

$$
u_{m} \rightarrow u \text { en } C\left([0, T] D\left(A^{\frac{\beta}{2}}\right)\right)
$$

Esta convergencia permite pasar al límite en el tercer término de (5.6).

Entonces, haciendo $m \rightarrow+\infty$ en (5.6) llegamos a

$$
\begin{gathered}
-\int_{0}^{T}\left(u^{\prime}(t)+\delta u(t), \psi^{\prime}(t)\right) d t+\int_{0}^{T}\left(A^{\frac{\alpha}{2}} u(t), A^{\frac{\alpha}{2}} \psi(t)\right) d t+ \\
+\int_{0}^{T}\left(p(t) A^{\frac{\beta}{2}} u(t), A^{\frac{\beta}{2}} \psi(t)\right) d t=\left(u_{1}+\delta u_{0}, \psi(0)\right)+\int_{0}^{T}(f(t), \psi(t)) d t
\end{gathered}
$$

para todo $\psi \in W^{\alpha}(0, T)$ tal que $\psi(T)=0$. Es decir, $u$ satisface $(5.1)$. 
Finalmente, las convergencias (5.4) y (5.5) implican que

$$
\lim _{m \rightarrow \infty} \max _{t \in[0, T]}\left(\left\|u_{m}^{\prime}(t)-u^{\prime}(t)\right\|^{2}+\left\|A^{\frac{\alpha}{2}} u_{m}(t)-A^{\frac{\alpha}{2}} u(t)\right\|^{2}\right)=0
$$

Notemos que

$$
\left\|u_{m}(0)-u(0)\right\|_{D\left(A^{\frac{a}{2}}\right)}^{2} \leq \max _{t \in[0, T]}\left\|A^{\frac{\alpha}{2}} u_{m}(t)-A^{\frac{\alpha}{2}} u(t)\right\|^{2}
$$

y

$$
\left\|u_{m}^{\prime}(0)-u^{\prime}(0)\right\|^{2} \leq \max _{t \in[0, T]}\left\|u_{m}^{\prime}(t)-u^{\prime}(t)\right\|^{2}
$$

Entonces, si $u_{0} \in D\left(A^{\frac{a}{2}}\right)$ y $u_{1} \in H$, cuando $m \rightarrow+\infty$ se tiene

$$
\begin{gathered}
u_{m}(0)=\sum_{k=1}^{m} g_{k}(0) w_{k}=\sum_{k=1}^{m}\left(u_{0}, w_{k}\right) w_{k}=u_{0 m} \rightarrow u_{0} \text { en } D\left(A^{\frac{\alpha}{2}}\right) \\
u_{m}^{\prime}(0)=\sum_{k=1}^{m} g_{k}^{\prime}(0) w_{k}=\sum_{k=1}^{m}\left(u_{1}, w_{k}\right) w_{k}=u_{1 m} \rightarrow u_{1} \text { en } H
\end{gathered}
$$

Entonces, tomando límite en (5.9) cuando $m \rightarrow+\infty$ y aplicando (5.8) y (5.11) probamos la condición inicial $u(0)=u_{0}$. Análogamente, de $(5.10),(5.12)$ y (5.8) probamos que $u^{\prime}(0)=u_{1}$.

Por tanto $u$ es una solución débil de (4.1). La unicidad de la solución débil puede obtenerse mediante el Método de Vishik-Ladyzhenskaya.

Finalmente, multiplicando la ecuación (4.3) por $g_{k}^{\prime}(t)$ y sumando desde $k=0$ hasta $k=m$ tenemos

$$
\left(u_{m}^{\prime \prime}(t)+\delta u_{m}^{\prime}(t)+A^{\alpha} u_{m}(t)+p(t) A^{\beta} u_{m}(t), u_{m}^{\prime}(t)\right)=\left(f(t), u_{m}^{\prime}(t)\right)
$$

es decir

$$
\frac{1}{2} \frac{d}{d t}\left(\left\|u_{m}^{\prime}\right\|^{2}+\left\|\frac{\alpha}{A^{2}} u\right\|^{2}\right)+\delta\left\|u_{m}^{\prime}\right\|^{2}+p(t)\left(A^{\beta} u_{m}, u_{m}^{\prime}\right)=\left(f(t), u_{m}^{\prime}\right)
$$

entonces, haciendo $m \rightarrow+\infty$ y aplicando las convergencias (5.4) y (5.5) obtenemos

$$
\frac{1 d}{2 d t}\left(\left\|u^{\prime}\right\|^{2}+\left\|A^{\frac{\alpha}{2}} u\right\|^{2}\right)+\delta\left\|u^{\prime}\right\|^{2}+p(t)\left(A^{\beta} u, u^{\prime}\right)=\left(f(t), u^{\prime}\right)
$$

Podemos resumir lo anterior en el siguiente

Teorema 5.2 Sean $T, \delta, \alpha$ y $\beta$ números reales positivos, con $\alpha \geq 2 \beta$. Sea $p$ una función continua sobre $[0, T]$ y $f \in L^{\infty}(0, T ; H)$. Entonces, si $u_{0} \in D\left(A^{\frac{\alpha}{2}}\right)$ y $u_{1} \in H$, el problema de 
valor inicial abstracto (4.1) tiene una única solución débil $u$ en la clase

$$
u \in C\left([0, T] ; D\left(A^{\frac{\alpha}{2}}\right)\right) \cap C^{l}([0, T] ; H)
$$

y satisface la siguiente identidad de energia

$$
\frac{1}{2} \frac{d}{d t}\left(\left\|u^{\prime}\right\|^{2}+\left\|A^{\frac{\alpha}{2}} u\right\|^{2}\right)+\delta\left\|u^{\prime}\right\|^{2}+p(t)\left(A^{\beta} u, u^{\prime}\right)=\left(f(t), u^{\prime}\right)
$$

Observación 5.3 (Dependencia Continua). La solución aproximada $u_{m}$ definida en (4.2) es tal que cada una de las funciones $g_{k}$ es solución de (4.4), entonces de acuerdo al Teorema 3.3, satisface la desigualdad (3.13), i.e.,

$$
g_{k}^{\prime}(t)^{2}+\lambda_{k}^{\alpha} g_{k}(t)^{2} \leq\left(g_{k}^{\prime}(0)^{2}+\lambda_{k}^{\alpha} g_{k}(0)^{2}+\frac{1}{2 \delta} \int_{0}^{t}\left(f(t), w_{k}\right)^{2} d s\right) \exp \left(\lambda_{k}^{\beta-\frac{\alpha}{2}} m_{0} t\right)
$$

Sumando en $k$ tenemos

$$
\left\|u_{m}^{\prime}(t)\right\|^{2}+\left\|A^{\frac{\alpha}{2}} u_{m}(t)\right\|^{2} \leq\left(\left\|u_{1 m}\right\|^{2}+\left\|u_{0 m}\right\|_{D\left(A^{\frac{\alpha}{2}}\right)}^{2}+\frac{1}{2 \delta}\|f\|_{L^{2}(0, T ; H)}^{2}\right) \exp (\Lambda T)
$$

donde $\Lambda=\lambda_{1}^{\beta-\frac{\alpha}{2}} m_{0}$ y $\lambda_{1}$ es el primer autovalor de $A$. Luego, haciendo $m \rightarrow+\infty$ y gracias a (5.8), (5.11) y (5.12) tenemos

$$
\left\|u^{t}(t)\right\|^{2}+\left\|A^{\frac{\alpha}{2}} u(t)\right\|^{2} \leq C\left(\left\|u_{1}\right\|^{2}+\left\|u_{0}\right\|_{D\left(A^{2}\right)}^{2}+\|f\|_{L^{\infty}(0, T ; H)}^{2}\right)
$$

para alguna constante $C>0$. Esta desigualdad prueba la dependencia continua de $u$ y su derivada en relación a $f$ y los datos iniciales.

\section{Comentarios Finales}

\subsection{Sobre la condición $\alpha \geq 2 \beta$}

La condición $\alpha \geq 2 \beta$ en el Teorema 5.2 es necesaria para hacer el pasaje al límite en el tercer término (5.6) y para acotar el término exponencial en (5.14). Si por el contrario, la condición fuese $\alpha<\beta$ entonces el Teorema 5.2 continúa siendo válido desde que consideremos la condición inicial $u_{0} \in D\left(A^{\frac{\beta}{2}}\right)$. La demostración es básicamente la misma, con las modificaciones naturales, por ejemplo en (5.2) reemplazamos $A^{\frac{\alpha}{2}}$ por $A^{\frac{\beta}{2}}$. Sin embargo, la prueba de la dependencia continua debe ser hecha de otra manera. 


\subsection{Un caso particular}

Si $A$ es el operador $-\Delta$ con dominio $D(-\Delta)=H_{0}^{1}(\Omega) \cap H^{2}(\Omega)$ siendo $\Omega \subset \mathbb{R}^{n}$ de clase $C^{2}$ y $\alpha=2, \beta=1$ tenemos el sistema linealizado de Petrovsky

$$
\left\{\begin{array}{l}
\frac{d^{2} u}{d t^{2}}+\delta \frac{d u}{d t}+\Delta^{2} u-p(t) \Delta u=f \\
u(0)=u_{0}, \frac{d u}{d t}(0)=u_{1}
\end{array}\right.
$$

el cual, de acuerdo al Teorema 5.2 posee una única solución débil $u$ en la clase

$$
u \in C\left([0, T] ; H_{0}^{1}(\Omega) \cap H^{2}(\Omega)\right) \cap C^{1}\left([0, T] ; L^{2}(\Omega)\right)
$$

desde que $u_{0} \in H_{0}^{\mathrm{l}}(\Omega) \cap H^{2}(\Omega)$ y $u_{1} \in L^{2}(\Omega)$.

\subsection{Otros problemas}

Las técnicas presentadas para la solución de (4.1) pueden ser aplicadas a la solución de otros problemas.

En (4.1), el término $\delta \frac{d u}{d t}$ representa la disipación del sistema. Cuando $A=-\Delta$ y $\Omega$ es un dominio acotado de $\mathbb{R}^{n}$, (4.1) toma la forma

$$
\frac{d^{2} u}{d t^{2}}+\delta \frac{d u}{d t}+(-\Delta)^{\alpha} u+p(t)(-\Delta)^{\beta} u=f, \text { en } \Omega \times(0, T)
$$

Esta ecuación generaliza la de las vibraciones de una placa $\Omega$. En este caso, la disipación actúa sobre toda la placa $\Omega$. Un problema a considerar es el de las vibraciones de una placa con disipación localizada, es decir,

$$
\frac{d^{2} u}{d t^{2}}+\chi_{\omega} \frac{d u}{d t}+(-\Delta)^{\alpha} u+p(t)(-\Delta)^{\beta} u=f, \text { en } \Omega \times(0, T)
$$

donde $\omega$ es un subconjunto abierto de $\Omega$ y $\chi_{\omega}$ denota la función característica de $\omega$. En este caso el término disipativo $\chi_{\oplus} \frac{d u}{d t}$ indica que la disipación se da únicamente sobre $\omega$.

Siguiendo las ideas del presente trabajo podemos estudiar el problema con disipación fuerte

$$
\frac{d^{2} u}{d t^{2}}-\Delta\left(\frac{d u}{d t}\right)+(-\Delta)^{\alpha} u+p(t)(-\Delta)^{\beta} u=f, \text { en } \Omega \times(0, T)
$$


o más generalmente el problema abstracto

$$
\frac{d^{2} u}{d t^{2}}+A^{\gamma}\left(\frac{d u}{d t}\right)+A^{\alpha} u+p(t) A^{\beta} u=f
$$

Un último problema que apuntamos y que también puede abordarse siguiendo este artículo es el de la ecuación que describe las vibraciones de una placa elástica que se originan debido a la inercia rotacional, es decir,

$$
\frac{d^{2} u}{d t^{2}}-\Delta^{2}\left(\frac{d^{2} u}{d t^{2}}\right)+\Delta^{2} u=f
$$

Agradecimiento. El autor agradece al Profesor L. A. Medeiros por su constante apoyo y por las conversaciones que tuvimos en relación al presente artículo.

\section{REFERENCIAS BIBLIOGRÁFICAS}

[1] BOUTET DE MONVEL L., CHUESHOV I. D. Non-linear oscillations of a plate in a flow gas. C. R. Acad. Sci. Paris. Ser. I, Vol. 322, pp 1002 - 1006, (1996).

[2] CABANILLAS ZANNINI, V. R., Remarks on a Linearized Petrovsky Model: Regular Solutions and its Associated Family of Ordinary Differential Equations. (2005). En vías de publicación.

[3] CHUESHOV, I., Introducction to the Theory of Infinite - Dimensional Dissipatives Systems. Acta, Kharkov, (2002).

[4] CODDINGTON, E., LEVINSON, N., Theory of Ordinary Differential Equations, McGrawHill, New York, (1955).

[5] ZEIDLER, E., Nonlinear Functional Analysis, Vol. II / B, Springer - Verlag. (1986). 